\title{
Cross-education: evidências, mecanismos, implicações para a reabilitação e aplicações práticas
}

\section{Cross-education: evidences, mechanisms, implications for practical rehabilitation and application}

\author{
Kelly Cristina de Mello Moraes', Ronei Silveira Pinto'
}

\section{Resumo}

O treinamento de força gera adaptações no sistema neuromuscular. Nesta perspectiva, o "cross-education" surge como um fenômeno caracterizado por adaptações neurais e pelo aumento da capacidade de geração de força voluntária do membro oposto ao não treinado, que ocorre como resultado do treinamento de força unilateral. Sendo assim, o presente estudo tem como objetivo fornecer informações sobre o fenômeno cross-education, apresentando suas evidências, mecanismos, implicações para a reabilitação e aplicações práticas. Neste trabalho, foi realizado um levantamento bibliográfico sobre o cross-education nas bases de dados disponíveis na Internet. As fontes de informações eletrônicas foram acessadas nas bases de dados do Pubmed, periódicos da CAPES e Lilacs. Para a busca de informações foram utilizadas as seguintes expressões: muscle strength, unilateral exercise, cross-education. A pesquisa inclui trabalhos publicados entre os anos de 1997 a 2015. Os estudos mostraram que o cross-education é caracterizado por adaptações neurais assim como por alterações no SNC que possibilitam a transferência de força para o membro contralateral, imobilizado ou não imobilizado. Conforme os resultados, este fenômeno parece ser importante para a reabilitação, à medida que ao realizarmos o treinamento de um membro isolado, podemos obter repercussões no membro contralateral. No entanto, mais pesquisas são necessárias para investigar as evidências e os mecanismos relacionados ao cross-education, assim como seus efeitos crônicos. Consequentemente, estudos complementares sobre esse fenômeno serão importantes para analisar a reabilitação de indivíduos com alguma lesão dos membros e as suas diversas aplicações práticas.

\section{Palavras-chave}

Força muscular; Exercício unilateral; Educação cruzada.

\begin{abstract}
Strength Training generates adaptations in neuromuscular system. Within this perspective "cross-education" emerges as a phenomenon featured by neural adaptations and by the increase of the capacity to generate volunteer strength of the opposite member to the non-trained one that occurs as a result of the unilateral strength training. Hence, the present study has as its objective provide information about the cross-education phenomenon, presenting its evidences, mechanisms, implications for practical rehabilitation and applications. In this paper a bibliographic survey about the cross-education has been made with data available on the internet. The electronic sources of information were accessed in the data base from Pubmed, from CAPES journals and Lilacs. For the search of information the following expressions were used: muscle strength, unilateral exercise, cross-education. The research includes papers published between the years of 1997 to 2015. Studies showed that cross-education is featured by neural adaptations so as by alterations at the SNC that enable the transference of strength to the contralateral limb, fixed or not fixed. According to its results, this phenomenon seems to be essential to rehabilitation, because as we train the static member we can obtain repercussions in the subordinate member. However, more research is necessary to investigate evidences and the mechanisms related to cross-education, as well as its chronic effects. As a result, complementary studies about this phenomenon will be important to analyze the rehabilitation of people with any member injuries and its many practical applications.
\end{abstract}

Keywords

Muscle Strength; Unilateral Exercise; Cross-Education.

\section{Introdução}

Uma vez que o sistema nervoso está envolvido na adaptação muscular, o treinamento de força unilateral também pode afetar os músculos do membro contralateral, de maneira que a adaptação no sistema neuromuscular pode ser observada no lado destreinado ${ }^{1}$. Embora ainda não sejam conhecidos os mecanismos envolvidos nesta adaptação, tem sido repor- 
tado na literatura o efeito do treinamento muscular de um membro (unilateral) no membro contralateral ${ }^{2-4}$.

O cross-education ou "Educação Cruzada" parece ser uma forma de adaptação neural ${ }^{1}$, em que ocorre a melhoria de desempenho (força, execução, habilidade, resistência) do membro não treinado (contralateral) após um período de prática unilateral do membro contralateral homólogo ${ }^{5-7}$, provavelmente induzido por mudanças no padrão de ativação de seus músculos, sem que haja alterações na sua morfologia ${ }^{1}$.

Nesta perspectiva, estudos demonstraram que o treinamento de força unilateral pode induzir de 5 a $25 \%$ o ganho de força no membro contralateral homólogo ${ }^{8}$. Recentemente, uma meta-análise com 13 estudos randomizados concluiu que a força unilateral produz um efeito pequeno, mas estatisticamente significativo sobre a força dos músculos homólogos no lado contralateral (aumento de $8 \%$ da força inicial do membro não treinado) ${ }^{9}$.

Por outro lado, alguns trabalhos não encontraram alterações no tamanho do músculo após duas ${ }^{10}$ e três semanas ${ }^{11}$ de imobilização. Esse fato foi verificado também no estudo de Fronteira et al. ${ }^{12}$ que, ao verificar o tamanho do músculo, a força muscular e o tamanho da fibra muscular durante 2 semanas de exercícios resistidos em 7 jovens e 7 idosos observaram que, após duas semanas, a força muscular aumentou em ambos os grupos enquanto o tamanho da fibra muscular não foi alterado.

Apesar da relevância clínica e científica do cross-education, é importante que haja uma melhor compreensão do seu papel para a adaptação neural, a fim de maximizar seus benefícios terapêuticos da reabilitação clínica $^{13}$. O estudo de Sariyildiz et al. ${ }^{14}$, mostrou que 6 semanas de estímulo de contração excêntrica dos flexores do punho no membro dominante pode causar um aumento na força muscular do extensores do punho contralateral em homens adultos saudáveis. Os resultados também sugerem que esta educação cruzada não se limita ao músculo homólogo contralateral inexperiente e que o ganho de força muscular pode também ser observado no membro contralateral antagonista. A perda de força muscular e atrofia das extremidades imobilizadas pode ser prevenida por estimulação elétrica unilateral em pacientes com imobilização unilateral. Adicionalmente, um estudo de Farthing et al. ${ }^{15}$ mostraram que o treinamento de força isométrico do membro saudável pode atenuar a perda de força no membro imobilizado durante um período de 3 semanas de imobilização unilateral. O aumento da força dos músculos homólogos e antagonistas contralaterais não treinados por contralateral estimulado contração excêntrica pode propiciar um benefícios terapêuticos em pacientes com lesões unilaterais.

Por conseguinte, torna-se importante o entendimento sobre as alterações neuromusculares e de seus mecanismos durante a fase inicial de treinamento de força para a prescrição de exercícios, visto que é nesta fase que ocorrem as adaptações neurais por meio da ativação voluntária máxima dos músculos treinados ${ }^{16}$. Sendo assim, o presente artigo tem como objetivo fornecer informações sobre o fenômeno cross-education, apresentando suas evidências, mecanismos, implicações para a reabilitação e aplicações práticas.

\section{Evidências e mecanismos do cross-education}

O cross-education foi observado pela primeira vez em $1894^{17}$, sendo então caracterizado por adaptações neurais e pelo aumento da capacidade de geração de força voluntária do membro oposto ao não treinado, que ocorre como resultado do treinamento de força unilateral.

Autores sugeriram que a adaptação neural parece ocorrer em vários locais dentro 
do Sistema Nervoso Central (SNC) e contribui para o efeito do cross-education ${ }^{1}$. Desta forma, o ganho de força parece ser específico para o músculo homólogo, sendo que estudos demonstram que a magnitude da transferência de força para o membro não treinado homólogo é proporcional ao ganho de força no membro treinado ${ }^{1}$.

A partir de sua descoberta, inúmeros estudos passaram a procurar evidências para apoiar a existência do cross-education. Estas têm sido observadas em resposta a diferentes tipos de treinamento (isométrico, concêntrico ou excêntrico) ${ }^{18}$, em grandes ou pequenos grupos musculares, em diferentes formas de contração (estática e dinâmica), tanto nos membros inferiores como nos superiores e tanto no membro dominante como também no não dominante ${ }^{19}$. Nesse contexto, em relação ao tipo de treinamento, a literatura tem reportado que as contrações excêntricas são capazes de produzir três vezes mais força do que contrações concêntricas ou isométricas $^{20}$, sendo importantes os efeitos do cross-education por meio de um trabalho excêntrico para o desenvolvimento da força muscular durante a reabilitação.

Por outro lado, Zijdewind e Kernell ${ }^{21}$ mostraram que contrações voluntárias máximas (CVM) em um grupo muscular podem induzir uma coativação involuntária muscular no membro homólogo contralateral. Esta coativação involuntária do músculo homólogo contralateral é discutida como um mecanismo potencial para o fenômeno do cross-education ${ }^{22}$. Além disso, pesquisas revelam que o ganho de força obtido a partir do efeito induzido pelo cross-education pode ser mantido durante o período de destreinamento ${ }^{23}$, em que ocorrem diminuições da contração voluntária máxima isométrica e não há alterações da área de secção transversa muscular no membro contralateral não treinado ${ }^{23}$. Assim, a força muscular adquirida pelo cross-education pode ser explicada por fatores neurais centrais, não somente durante o treinamento, mas também durante o período de destreinamento ${ }^{24}$. $\mathrm{Na}$ tentativa de estabelecer se a atividade gerada durante o treinamento unilateral impulsiona adaptações simultâneas em ambos os hemisférios cerebrais que são suficientes para aumentar a capacidade funcional do membro não treinado, é necessário, portanto, avaliar a totalidade de vias neurais e mecanismos que podem desempenhar um papel causal. Reconhecendo que, todo o controle de tarefas é obtido através da modulação equilibrada de processos inibitórios e facilitadores, torna-se importante considerar se variações específicas neste equilíbrio podem surgir através do treinamento e exercer um efeito funcional sobre movimentos do membro oposto 25 .

Durante o período de destreinamento, podem ocorrer diminuições da contração voluntária máxima (CVM) isométrica e IEMG sem alterações na área de secção transversa do músculo (CSA) no membro contralateral não treinado ${ }^{22}$. Esta pesquisa não relatou nenhuma alteração na CSA muscular no membro contralate$\mathrm{ral}^{22}$, no entanto, Housh et al. ${ }^{26}$, observaram uma tendência de aumento muscular CSA no lado contralateral após 8 semanas de treinamento de força unilateral.

O fenômeno cross-education, é enfatizado a partir de adaptações neurais que são mudanças na coordenação e no aprendizado e facilitam a ativação muscular durante um treinamento de força, sendo indiretamente verificadas por eletromiografia ${ }^{27}$. Envolvem ajustes no sistema nervoso para promover a ativação muscular máxima (eficiência no recrutamento das fibras musculares, melhora da ativação neural e diminuição da coativação da musculatura agonista) ${ }^{4}$. Um estudo que comprova este incremento foi realizado com sujeitos durante seis semanas de treinamento unilateral de flexores de cotovelo, tendo isso reportado um aumento de $7 \%$ de força no membro contralateral (não treinado) ${ }^{28}$. Já, em uma meta-análise composta por 16 estudos com duração de 15 a 48 sessões de treinamento, os au- 
tores revelaram que o tamanho do efeito de treinamento de força contralateral é $8 \%$ da força inicial, ou cerca da metade do aumento da força do lado treinado ${ }^{1}$.

Além disso, a ativação neural pode ser alterada pelo número de unidades motoras recrutadas, pela frequência de disparo de cada unidade motora, pela coordenação entre os músculos sinérgicos e pelos reflexos inibitórios ${ }^{3}$. Durante as fases iniciais de um treinamento de força, há o predomínio de adaptações neurais ${ }^{29}$. Em vista disso, muitas pesquisas sobre treinamento foram realizadas relativamente em um período de 20 semanas. É nas primeiras semanas de treinamento que, os participantes que não apresentam experiência prévia em treinamento, geralmente, demonstram um rápido aumento de força muscular ${ }^{8}$. Porém, essas adaptações iniciais ocorrem em decorrência de qualquer tipo de treinamento de força, visto que as adaptações neurais que ocorrem nas primeiras semanas de treinamento parecem promover um aumento mais rapidamente de força muscular.

Acredita-se que o cross-education ocorra como resultado de adaptações neurais, porém, seus mecanismos exatos exigem uma investigação mais aprofundada, à medida que os estudos verificados na literatura não conseguiram determinar o nível de contribuição a partir de locais específicos dentro do $\mathrm{SNC}^{18}$.

No entanto, os mecanismos do cross-education podem desempenhar um papel importante na manutenção da função neural durante períodos de imobilização ${ }^{18}$. Porém, alguns achados sugerem que a adaptação neural no membro não treinado ocorra predominantemente em nível cortical e que o treinamento unilateral pode induzir alterações nas estruturas neurais que inervam o membro contralateral não treinado, que pode atuar como um mecanismo responsável pelo ganho de força no membro não treinado ${ }^{18}$.

\section{Implicações para a reabilitação e aplicações prática do cross-education}

Os efeitos induzidos pelo cross-education parecem ser importantes para a reabilitação neuromuscular, visto que a realização de um treinamento de força unilateral pode constituir uma oportunidade para evitar a perda de força e atrofia por meio de transferência de força para o músculo inativo ${ }^{18}$.

Em um estudo foi observado que a força muscular das fibras tipo I, IIa e IIb do quadríceps dos indivíduos foi reduzida em 13, 10 e 10\% respectivamente com três meses de imobilização ${ }^{30}$. Paulatinamente, os mesmos autores revelaram que os indivíduos que não podem movimentar um membro ou que neste membro não há funcionalidade da inervação muscular, podem se beneficiar de exercício contralateral, possibilitando assim a diminuição da atrofia muscular, a manutenção da coordenação motora e o aumento da força muscular do membro afetado ${ }^{30}$.

Paralelo a isso, é importante verificar este efeito durante o desuso, visto que a maior perda de força muscular parece ocorrer nas duas primeiras semanas em que um segmento é subutilizado ${ }^{30}$. Neste contexto, autores realizaram recentemente uma pesquisa com o cross-education, tendo sido verificado que o treinamento de força no membro não imobilizado atenuou a perda de força e o tamanho do músculo do membro imobilizado durante um período de três semanas. Este estudo sugere que o treinamento de força no membro não imobilizado fornece um efeito de manutenção para o membro imobilizado ${ }^{30}$. Da mesma forma, foi observado também que pacientes que não podem exercer ativamente um membro podem se beneficiar com o cross-education devido à diminuição da atrofia muscular, à manutenção da coordenação motora, e ao aumento da força muscular do membro afetado ${ }^{8}$.

Essas considerações são importantes à medida que a fraqueza muscular é uma causa comum de recuperação funcional após lesões e doenças, evidenciando que o 
treinamento de força é parte integrante da reabilitação e de programas de promoção da saúde em ambientes comunitários ${ }^{2}$. Segundo estes autores, o cross-education apresenta relevância para a reabilitação de pacientes sem condições de utilizar um membro e se, ao exercitar o membro saudável existe a possibilidade de fortalecer o membro ferido ou doente, poderá assim minimizar as complicações causadas pelo desuso e maximizar a eficácia da reabilitação após a cura da lesão².

Até o momento, dois estudos tem tentado aplicar o para manter a força e a espessura muscular em indivíduos com membros imobilizados ${ }^{31}$. Ambos os estudos encontraram que a força e a espessura muscular do membro imobilizado foram mantidas por um período de três semanas pelo grupo experimental que realizaram treinamento de força unilateral. Os participantes do grupo controle que foram submetidos à imobilização e não treinaram o membro oposto, obtiveram uma redução na força e espessura muscular, indicando que os resultados positivos foram diretamente gerados pelo cross-education. De acordo com estes estudos, o sucesso na manutenção da força muscular e da espessura muscular em indivíduos imobilizados fornece perspectivas promissoras para a recuperação mais efetiva, reduzindo assim o impacto da imobilização ${ }^{31}$. Para os atletas, a perda de força associada com a imobilização muitas vezes reduz o desempenho e exige ao indivíduo se submeter a um período de reabilitação ${ }^{19}$.

Em outros casos, como o de pacientes idosos que necessitam de imobilização, muitas vezes apresentam maiores dificuldades de recuperar a função muscular, prejudicando assim sua independência e qualidade de vida ${ }^{32}$. Em qualquer caso, a manutenção da função muscular durante a imobilização irá fornecer resultados positivos para os pacientes. Neste sentido, os resultados de estudos sobre os efeitos relacionados ao cross-education sugerem que a transferência de força pode desempenhar um papel vital para a recuperação de determinados pacientes ${ }^{19}$. Neste caso, uma pesquisa descobriu que o treinamento de força no membro não imobilizado proporcionou uma manutenção da força no membro imobilizado após três semanas de imobilização ${ }^{33}$. Portanto, em todos os casos citados acima, o tema cross-education possui certa relevância e, principalmente, tem se expandido no campo da reabilitação para o tratamento de indivíduos imobilizados ou que não possam por determinada circunstância movimentar o membro com lesão.

Por conseguinte, a aplicação de um treinamento de força unilateral parece relevante para a recuperação e desempenho do membro homólogo inativo durante a imobilização. Desta forma, futuras pesquisas envolvendo os efeitos dos mecanismos do cross-education em um ambiente clínico são necessárias para determinar as respostas fisiológicas e neurológicas mais complexas dos pacientes. É possível que o treinamento de força unilateral possa ter implicações clínicas para pacientes com distúrbios neurológicos.

\section{Métodos}

O presente estudo é uma revisão de literatura com a realização de um levantamento de artigos originais e revisões publicadas em revistas internacionais e nacionais.

Neste trabalho foi realizado um levantamento bibliográfico para capturar o maior número de citações relevantes possíveis sobre o fenômeno cross-education nas bases de dados informatizadas disponíveis na Internet. As fontes de informações eletrônicas foram acessadas nas bases de dados do Pubmed, periódicos da CAPES e Lilacs.

Para a busca de informações foram utilizadas as seguintes expressões na língua inglesa: cross-education, cross effect, strenght training, contralateral adaptations, unilateral exercise. 
A pesquisa inclui trabalhos publicados entre os anos de 1997 a 2015. Para a análise dos resultados, foram utilizados como critérios de inclusão os estudos que se enquadravam nos seguintes aspectos: 1) estudos relacionados às evidências e os mecanismos do cross-education; 2) estudos referentes à reabilitação e direcionados às aplicações práticas do cross-education.

Dentre o total de artigos, foram utilizados 13 estudos que se enquadravam nos critérios descritos acima.

\section{Resultados}

O cross-education é caracterizado primeiramente por adaptações neuromusculares que envolvem ajustes no SNC para a ativação máxima muscular ${ }^{4}$ e se desenvolvem nas etapas iniciais do treinamento (4-6 semanas) ${ }^{3}$.

O quadro 1 representa as evidências e os mecanismos do cross-education, obtendo os estudos encontrados sobre as adaptações neuromusculares provocadas por este fenômeno, assim como os mecanismos que ocorrem no SNC e as evidências na literatura até os dias atuais.

QUADRO 1 - Estudos que avaliaram as evidências e os mecanismos do cross-education.

\begin{tabular}{|c|c|c|c|}
\hline Estudo & Amostra & Treinamento & Conclusões \\
\hline Shima et al. ${ }^{24}$ & $\begin{array}{l}15 \text { homens adultos } \\
26,2 \pm 4,6 \text { anos }\end{array}$ & $\begin{array}{l}6 \text { semanas } \\
3 \text { séries de } 10-12 \text { repetições } \\
70-75 \% \text { de } 1 \text { RM }\end{array}$ & $\begin{array}{l}\text { O cross-education pode ser explicado por adaptações neurais } \\
\text { durante o treinamento e o destreinamento. }\end{array}$ \\
\hline Fimland et al. ${ }^{15}$ & 26 (homens) & $\begin{array}{l}4 \text { semanas } \\
6 \text { séries } 6 \text { CVM } \\
40-70 \% \text { CVM }\end{array}$ & $\begin{array}{l}\text { O cross-education contribui para o aumento da unidade } \\
\text { neural dos músculos agonistas. }\end{array}$ \\
\hline Farthing et al. ${ }^{5}$ & 23 mulheres & $\begin{array}{l}6 \text { semanas } \\
4 \times \text { semana }\end{array}$ & $\begin{array}{l}\text { O cross-education pode ser controlado } \\
\text { por adaptações do córtex sensoriomotor. }\end{array}$ \\
\hline Lee et al. ${ }^{37}$ & 20 homens & 4 semanas & $\begin{array}{l}\text { Melhorias na unidade motora cortical } \\
\text { contribuem para os efeitos do treinamento de } \\
\text { força contralateral. }\end{array}$ \\
\hline Adamsom et al. ${ }^{40}$ & 10 mulheres & $\begin{array}{l}8 \text { semanas } \\
3 x \text { por semana } \\
5 \text { séries de } 5 \text { RM }\end{array}$ & $\begin{array}{l}\text { O treinamento de força pode promover o desenvolvimento de } \\
\text { força máxima e rápida no membro contralateral homólogo. }\end{array}$ \\
\hline Munn et al. ${ }^{28}$ & 115 (mulheres e homens) & $\begin{array}{l}18 \text { sessões } \\
6-8 \text { repetições } 80 \% \text { (1RM) }\end{array}$ & $\begin{array}{l}\text { A força muscular unilateral produz aumento de força } \\
\text { contralateral. }\end{array}$ \\
\hline Ehsani et al. ${ }^{38}$ & $\begin{array}{l}12 \text { adultos ( } 24-32 \text { anos); } \\
12 \text { idosos (64-79 anos). }\end{array}$ & $\begin{array}{l}\text { Exercício isométrico; } \\
3 \text { séries de } 10 \text { repetições; } \\
60-70 \% \text { da força máxima; } \\
2 \text { semanas; } \\
3 x \text { por semana. }\end{array}$ & $\begin{array}{l}\text { A força muscular aumentou nos membros destreinados, o que } \\
\text { sugere que a capacidade de adaptação neuromuscular dos } \\
\text { idosos é adequada para casos de imobilidade do membro ou } \\
\text { de prejuízo unilateral. }\end{array}$ \\
\hline
\end{tabular}

RM: repetições máximas; CVM: Contração voluntária Máxima.

Foram encontrados um total de 6 estudos sobre o tema e, como principal achado, foi verificado que os efeitos provocados pelo fenômeno cross-education parecem ser gerados por adaptações neurais que contribuem para o aumento da força do membro contralateral ${ }^{34}$.

Por outro lado, o cross-education apresenta relevância para a imobilização, sendo importante para a reabilitação de lesões e assim apresenta aplicações práticas de protocolos de treinamento de força. Então, o quadro 2 revela estudos relacionados com a reabilitação e as aplicações práticas baseadas nos benefícios específicos provocados pelo cross-education.

Neste quadro, estão representados um total de 7 estudos que esclarecem os efeitos do cross-education para indivíduos com lesões nos membros. Os dados demonstrados no quadro 2 apresentaram como principal achado que o treinamento 
unilateral produz benefícios para a reabilitação de lesões ${ }^{35}$.

QUADRO 2 - Estudos que avaliaram as implicações para a reabilitação e as aplicações práticas do cross-education.

\begin{tabular}{|c|c|c|c|}
\hline Estudo & Amostra & Treinamento & Conclusões \\
\hline Timothy et al..$^{35}$ & 7 homens e 11 mulheres & Movimentos balísticos & $\begin{array}{l}\text { O treinamento de força pode induzir implicações clínicas } \\
\text { importantes para a reabilitação de movimentos. }\end{array}$ \\
\hline Magnus et al..$^{31}$ & 25 (homens e mulheres) & $\begin{array}{l}4 \text { semanas com membro } \\
\text { imobilizado }\end{array}$ & $\begin{array}{l}\text { O treinamento de força do membro não } \\
\text { imobilizado proporciona aumento da força do } \\
\text { membro imobilizado. }\end{array}$ \\
\hline Farthing et al..$^{30}$ & $\begin{array}{l}30 \text { (homens e mulheres) } \\
3 \text { grupos }\end{array}$ & $\begin{array}{l}15 \text { sessões } \\
\text { 3-6 séries } \\
8 \text { repetições }\end{array}$ & $\begin{array}{l}\text { O treinamento unilateral proporciona benefícios para o } \\
\text { membro imobilizado. }\end{array}$ \\
\hline Farthing \& Chilibeck. ${ }^{36}$ & $\begin{array}{l}26 \text { (homens e mulheres) } \\
2 \text { grupos }\end{array}$ & $\begin{array}{l}8 \text { semanas } \\
3 x \text { por semana } \\
\text { Treinamento excêntrico } \\
\text { unilateral dos flexores do } \\
\text { cotovelo. }\end{array}$ & $\begin{array}{l}\text { O treinamento excêntrico unilateral de alta velocidade parece } \\
\text { promover adaptações no membro contralateral homólogo. } \\
\text { Estes resultados possuem implicações para a reabilitação de } \\
\text { lesões. }\end{array}$ \\
\hline Magnus et al..$^{41}$ & $\begin{array}{l}39 \text { mulheres com mais de } \\
50 \text { anos de idade. }\end{array}$ & $\begin{array}{l}3 \times \text { por semana } \\
26 \text { semanas }\end{array}$ & $\begin{array}{l}\text { O treinamento de força para o membro não fraturado foi } \\
\text { associado à melhora da força no membro com fratura do } \\
\text { rádio em } 12 \text { semanas após a fratura. }\end{array}$ \\
\hline $\begin{array}{l}\text { Lepley LK, Palmieri- } \\
\text { Smith RM. }{ }^{43}\end{array}$ & 18 indivíduos & $\begin{array}{l}3 x \text { por semana } \\
8 \text { semanas } \\
\text { Treinamento excêntrico }\end{array}$ & $\begin{array}{l}\text { Os ganhos de força ocorreram por causa da atividade neural } \\
\text { melhorada, sendo que os efeitos do cross-education podem } \\
\text { ser observados em programas de reabilitação. }\end{array}$ \\
\hline
\end{tabular}

Sendo assim, os 13 estudos enquadrados no tema desta pesquisa, explícitos no quadro 1 e no quadro 2, revelaram os efeitos gerados pelo treinamento de força no membro contralateral, as adaptações neurais, os benefícios para a reabilitação de lesões, os tipos de contrações musculares que geram o aumento de força rápida e máxima por meio do cross-education e as adaptações que ocorrem no período de treinamento e no destreino.

\section{Discussão}

Esta breve revisão destacou os diversos aspectos sobre as evidências, os mecanismos, as implicações para a reabilitação e as aplicações práticas do fenômeno cross-education. Embora o fenômeno tenha sido descrito há mais de um século, muitos estudos apresentam erros, o que impossibilita a definição exata da magnitude do efeito.

O cross-education é observado com a realização de um treinamento de força unilateral no membro oposto ao membro imobilizado e parece ser benéfico também para os efeitos neurais do membro oposto ao membro imobilizado durante o treinamento e o destreinamento ${ }^{23}$.

De acordo com alguns estudos, o cross-education proporciona um aumento das unidades neurais dos músculos agonistas e por este motivo, o número limitado de pesquisas sobre o tema demonstrou que é manifestado primeiramente por adaptações neurais ${ }^{36}$. Além disso, os mecanismos que ocorrem por meio deste fenômeno são gerados no SNC, principalmente no córtex sensoriomotor ${ }^{36}$.

Do mesmo modo, foram verificadas melhorias na unidade motora cortical que contribuem aos efeitos do treinamento de força contralateral ${ }^{33}$. Lee et al.$^{37}$ e Munn et $a .^{28}$ refletiram em seus estudos que o treinamento de força unilateral é capaz de promover aumentos de força no membro contralateral homólogo. Segundo Lee et $\mathrm{al}^{37}$, isso ocorre devido aos mecanismos gerados nas unidades motoras corticais.

Além disso, o trabalho de Ehsani et al..$^{38}$ foi desenvolvido com 12 jovens (idades entre $28,25 \pm 3,11$ anos) e 12 idosos (idades entre $73.08 \pm 5,3$ anos), em que os 
sujeitos realizaram exercícios isométricos de flexão de cotovelo no lado do dominante, com 3 séries de 10 repetições da força máxima de $60-70 \%$ por duas semanas. Os resultados mostraram que, à curto prazo, houve aumento significativo de força nos membros treinados e destreinados em ambos os grupos $(\mathrm{p}<0,05)$. Não houve diferença significativa entre os dois grupos na taxa de aumento de força, tanto no membro superior e também no lado oposto $(p>0,05)$. Este aumento da força muscular nos membros destreinados sugere a capacidade de adaptação neuromuscular e, como os idosos são mais suscetíveis a dano, acidente vascular cerebral e trauma, o treinamento transversal pode ser benéfico em condições em que o membro afetado seja incapaz de exercer qualquer movimento.

A adaptação neuromuscular pode ser verificada também após uma única sessão de estimulação elétrica ${ }^{39}$ com trinta e seis homens adultos distribuídos aleatoriamente em grupo de estimulação remota $(\mathrm{RS} ; \mathrm{n}=18)$ ou grupo controle (CTL; $\mathrm{n}=18$ ). O grupo RS realizou um programa de estimulação elétrica de superfície no reto femoral da perna não dominante. Os indivíduos do grupo CTL ficaram 10 minutos sem executar qualquer treinamento. Antes e depois do programa de estimulação elétrica de superfície, a força isométrica, a eletromiografia (EMG) e a resposta mecanomiográfica (MMG) da perna dominante foram medidas em todos os indivíduos. A perna dominante do grupo RS mostrou um aumento significativo na força isométrica $(5,11 \%$; $\mathrm{p}<0,001)$ e na atividade EMG do músculo agonista (4,67\%; p<0,05), porém houve uma diminuição da atividade EMG dos músculos antagonistas $(-10,27 \%$; $\mathrm{p}<0,05)$. A atividade $M M G$ não obteve alterações. Não foram observadas mudanças significativas para o grupo CTL. Estes resultados indicam que uma sessão de estimulação elétrica no reto femoral parece capaz de melhorar a eficiência do membro inativo. Desta forma, os resultados abrem uma nova maneira de reabilitar lesões musculoesqueléticas, à medida que a força muscular pode ser melhorada através da eletroestimulação passiva no membro saudável.

As condições em que o cross-education se manifesta são capazes de promover o aumento da força máxima e da força rápida do membro contralateral ${ }^{40}$. Neste sentido, parece que quando realizado um treinamento unilateral excêntrico de alta velocidade, adaptações são geradas no membro contralateral, as quais são também importantes para a reabilitação de lesões ${ }^{34}$.

Desta forma, alguns autores refletiram sobre os benefícios provocados pelo cross-education para a reabilitação de indivíduos com lesões em algum membro e constataram a necessidade da aplicação de um treinamento de força unilateral no membro oposto ao membro imobilizado como forma de aumento da força muscular deste membro. Uma pesquisa com 26 indivíduos de ambos os sexos, que realizaram um treinamento unilateral excêntrico de flexores de cotovelo durante oito semanas, verificou que o cross-education ocorre com rápidas velocidades de contração e parece importante para a reabilitação de pessoas com lesões nos membros ${ }^{34}$.

Além disso, um estudo com 25 indivíduos de ambos os sexos, no qual ficaram com um membro imobilizado durante um período de 4 semanas, verificou que o treinamento de força realizado no membro não imobilizado é capaz de proporcionar aumento de força no membro imobilizado ${ }^{41}$. Corroborando a estes achados, Farthing et al..$^{30}$ realizaram um estudo com 30 indivíduos de ambos os sexos e concluíram que o treinamento de força unilateral promove benefícios para o membro imobilizado.

Adicionalmente, Magnus ${ }^{31}$ também analisou a importância do treinamento unilateral para a reabilitação clínica, sendo o primeiro estudo controlado e randomizado, para demonstrar que o treinamento unilateral no membro não fraturado pode beneficiar o membro homólogo fraturado. 
Por outro lado, atletas e treinadores estão interessados em programas de reabilitação para evitar a perda de força após lesões unilaterais, e também em acelerar o processo de recuperação ${ }^{42}$. Em vista disto, o estudo de Farthing $\&$ Chilibeck $^{36}$, esclareceram as evidências da prática do treinamento unilateral excêntrico para a reabilitação de lesões de membros.

Simplificando, esta revisão fornece detalhes da maioria dos estudos presentes na literatura sobre treinamento de força e o cross-education e referem ocorrer mecanismos no SNC assim como adaptações neurais durante o período de treinamento e destreinamento. Como foi visto anteriormente, foi constatada a relevância do cross-education para o ganho de força do membro contralateral em vários estudos, tornando-se possível a aplicação do treinamento de força unilateral em indivíduos imobilizados, principalmente em se tratando de reabilitação de lesões.

Em vista disso, como foi relatado pela primeira vez na década de 80, é um tema atual e são necessários mais estudos complementares para justificar a sua importância no contexto do treinamento de força que servirão para analisar a reabilitação de indivíduos com alguma lesão dos membros e as diversas aplicações práticas do cross-education, assim como seus efeitos crônicos.

\section{Agradecimentos}

À ESEF/ UFRGS pela oportunidade de realização deste trabalho. Ao professor orientador Ronei Pinto por todo apoio para a concretização desta pesquisa.

\section{Contribuição dos autores}

- Kelly: Concepção e projeto ou análise e interpretação dos dados e redação do artigo.

- Ronei: Revisão crítica relevante do conteúdo intelectual e aprovação final da versão a ser publicada.

\section{Referências}

1. Carroll TJ, Herbert RD, Munn J, Lee M, Gandevia SC. Contralateral effects of strength training: evidence and possible mechanisms. J Appl Physiol. 2006; 101: 1514-22.

2. Lee M, Carroll TJ. Cross Education Possible Mechanisms for the Contralateral Effects of Unilateral Resistance Training. Med Sci Sports Exerc. 2007; 37 (1): 1-14.

3. Housh TJ, Housh DJ, Weir JP, Weir LL. Effects of eccentric- only resistance training and detraining. Med Sci Sports Exerc. (1996b); 17:145-48.

4. Brentano MF, Pinto RS. Adaptações neurais ao treino de força. Rev Bras Ativ Fís Saúde. 2001; 6(3): 65-77.

5. Farthing JP; Borowsky R; Chilibeck PD; Binsted G; Sarty GE. Neuro-Physiological Adaptations Associated with Cross-Education of Strength. Brain Topogr. (2007) 20:77-88.

6. Enoka RM. Muscle strength and its development- new perspectives. Med Sci Sports Exerc.1988; 6: 146-68.

7. Sale DG. Neural adaptation to resistance training. Med Sci Sports Exerc. 1988; 20 (5): 135-45.

8. Zhou S. Cross education and neuromuscular adaptations during early stage of strength training. J Exerc Sci Fit. 2003; 1 (1): 54-60.

9. Munn J, Herbert RD, and Gandevia SC. Contralateral effects of unilateral resistance training: a meta-analysis. J Appl Physiol. 2004; 96: 1861-66.

10. Deschenes MR, Giles JA, McCoy RW, Volek JS, Gomez AL, Kraemer WJ. Neural factors account for strength decrements observed after shortterm muscle unloading. J Appl Physiol. 2002; 282: 578-583.

11. Kitahara A, Hamaoka T, Murase N, Homma T, Kurosawa Y, Ueda C et al. Deterioration of muscle function after 21-day forearm immobilization. Med Sci Sports Exerc. 2003; 35: 1697-702.

12. Frontera WR, Hughes VA, Krivickas LS, Kim SK, Foldvari M, Roubenoff R. Strengh training in older women: early and late changes in whole mus-cle and single cells. Muscle Nerve. 2003; 28(5):601-8. 
13. Zhou S. Chronic neural adaptations to unilateral exercise: mechanisms of cross education. Exerc Sport Sci Rev. 2000; 28: 177-84.

14. Sariyildiz, M, Karacan I, Rezvani A, Ergin O, Cidem M. Cross-education of muscle strength: cross-training effects are not confined to untrained contralateral homologous muscle. Scand J Med Sci Sports 2011: 21: e359-e364.

15. Farthing JP, Krentz JR, Magnus CRA. Strength training the free limb attenuates strength loss during unilateral limb immobilization. J Appl Physiol. 2009; 106: 830-36.

16. Hakkinen K, Komi PV. Electromyographic changes during strength training and detraining. Med Sci Sports Exerc. 1983; 15(6): 455-60.

17. Scripture EW, Smith TL, and Brown EM. On the education of muscular control and power. Studies Yale Psychol 1894; 2: 114-19.

18. Housh DJ, Housh TJ, Johnson GO, Chu W-K. Hypertrophic response to unilateral concentric isokinetic resistance training. J Appl Physiol. 1992; 73 (1): 65-70.

19. Panzer S, Schinowski D, Kohle D. Cross-Education and contralateral irradiation. J Hum Kinet. 2011; 27: 66-79.

20. Hendy AM, Spittle M, Kidgell DJ. Cross education and immobilisation: Mechanisms and implications for injury rehabilitation. Med Sci Sports Exerc. 2012; 15: 94-101.

21.Zijdewind, I, Kernell, D. Bilateral Interactions during contractions of intrinsic hand muscles.J Neurophysiol. 2001; 85: 1907-13.

22. Housh TJ, Housh DJ, Weir JP, Weir LL. Effects of eccentric- only resistance training and detraining. Med Sci Sports Exerc.1996b; 17:145-148.

23. Narici MV, Roi GS, Landoni L, Minetti AE, Cerretelli P. Changes in force, cross-sectional area and neural activation during strength training and detraining of the human quadriceps. J Appl Physiol.1989; 59:310-19.

24. Shima N, Ishida K, Katayama K, Morotome Y, Sato Y, Miyamura M. Cross education of muscular strength during unilateral resistance training and detraining. J Appl Physiol. (2002) 86: 287-94.

25. Ruddy KL and Carson RG. Neural pathways mediating cross education of motor function. Front Hum Neurosci. (2013) 7: 1-22.

26. Housh DJ, Housh TJ, Johnson GO, Chu WK (1992) Hypertrophic response to unilateral concentric isokinetic resistance training. JAppl Physiol 73:65-70.

27. Folland JP, Williams AG. The adaptations the strength training: morphological and neurological contributions to increased strength. Med Sci Sports Exerc. 2007; 37 (2): $145-68$.

28. Munn J, Herbert RD, Hancock MJ, Gandevia SC. Training with unilateral resistance exercise increases contralateral strength. J Appl Physiol. 2005; 99 (5): 1880-84.

29. Hortobágyi T, Dempsey L, Fraser D, Zheng D, Hamilton G, Lambert J et al. Changes in muscle strength, muscle fibre size and myofibrillar gene expression after immobilization and retraining in humans. J Appl Physiol. 2000; 524: 293-304.

30. Moritani MA, De Vries HA. Neural factors versus hypertrophy in the time course of muscle strength training. Arch Phys Med Rehabil. 1979; 58 (3):115-30.

31. Magnus CRA, Barss TS, Lanovaz JL, Farthing JP. Effects of cross-education on the muscle after a period of unilateral limb immobilization using a shoulder sling and swathe. J Appl Physiol. 2010; 109(6):1887-94.

32. Sattin RW, Lambert Huber DA, Devito CA, Rodriguez J, Ros A, Bacchelli S, et al.. The incidence of fall injury events among the elderly in a defined population. Am J Epidemiol. 1990; 131(6):1028-103.

33. Farthing JP, Krentz JR, Magnus CRA,Barss TS, Lanovaz JL, Cummine J et al. Changes in functional magnetic resonance imaging cortical activation with cross education to an immobilized limb. Med Sci Sports Exerc. 2011; 43:1394-405.

34. Fimland MS, Helgerud J, Solstad GM, Iversen FM, Hov J. Neural adaptations underlying cross-education after unilateral strength training. J Appl Physiol. 2009; 107:723-30.

35. Timothy J. Carroll, Michael Lee, Marlene Hsu and Janel Sayde. Unilateral practice of a ballistic movement causes bilateral increases in performance and corticospinal excitability. J Appl Physiol. 2008; 104: 1656 -64.

36. Farthing JP, Chilibeck PD. The effect of eccentric training at different velocities on crosseducation. J Appl Physiol. 2003; 89: 570-7. 
37. Lee M, Gandevia SC, Carroll TJ. Unilateral strength training increases voluntary activation of the opposite untrained limb.Clin Neurophysiol. 2009; 120: 802-8.

38. Ehsani F, Nodehi- Moghadam A, Ghandali H, Ahmadizade Z. The comparison of cross -education effect in young and elderly females from unilateral training of the elbow flexors. Med J Islam Repub Iran. 2014; 28:1-6.

39. Toca-Herrera J L, Gallach J E, Gomis M and Gonzalez LM. Cross-education after one session of unilateral surface electrical stimulation of the rectus femoris. Strength Cond Res 2008; 22(2):614-8.

40. Adamson M, MacQuaide N, Helgerud J, Hoff J, Kemi OJ. Unilateral arm strength training improves contralateral peak force and rate of force development. J Appl Physiol. 2008; 103: 553-59.

41. Magnus CR, Arnold C, Johnston G, Dal-Bello Haas V, Basran J, Krentz J et al. Education for Improving Strength and Mobility After Distal Radius Fractures: A Randomized Controlled Trial.Eur J Phys Med Rehabil. 2013; 94:1247-55.

42. Taniguchi $Y$. Lateral specificity in resistance training: the effect of bilateral and unilateral training. J Appl Physiol.1997; 75: 144-50.

43. Lepley LK, Palmieri- Smith RM. Cross-education strength and activation after eccentric exercise. J Athl Train. 2014 ;49(5):582-9.

ENDEREÇO PARA

CORRESPONDÊNCIA

KELLY CRISTINA DE MELLO MORAES

kelly.m.86@hotmail.com
Avenida Palmira Gobbi, 426 ap: 130

bloco 1D. Porto Alegre- RS.

CEP: 90250210
RECEBIDO $\quad 09 / 04 / 2015$

REVISADO 21/07/2015

$29 / 12 / 2015$

$15 / 04 / 2016$

APROVADO 26/05/2016 